\title{
Avaliação das alterações dentárias na maxila em pacientes submetidos à expansão rápida da maxila assistida cirurgicamente sem o envolvimento da sutura pterigomaxilar*
}

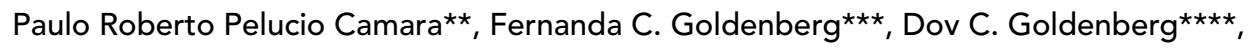

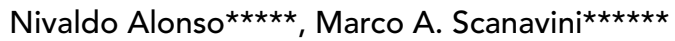

\section{Resumo}

Objetivo: avaliar as alterações nos arcos dentários superiores no sentido transversal e a quantidade de inclinações nos dentes de apoio do aparelho de expansão tipo Hyrax em pacientes submetidos à expansão rápida da maxila assistida cirurgicamente (ERMAC) e a efetividade dessa técnica cirúrgica utilizada. A amostra foi composta por 34 pares de modelos de gesso de 17 pacientes, sendo 6 do gênero masculino e 11 do gênero feminino. Métodos: as medidas foram realizadas em modelos de gesso por meio de medição das alterações nos planos vertical e transversal. O procedimento cirúrgico adotado foi uma osteotomia nas paredes laterais da maxila sem o envolvimento da lâmina pterigoide, osteotomia da espinha nasal à linha média dentária (incisivos centrais anteriores), separação da sutura palatina mediana por meio de cinzel e separação do septo nasal. O início das ativações ocorreu no terceiro dia pós-operatório, sendo uma pela manhã e uma à noite. Resultados: houve expansão significativa estatisticamente na região dos caninos, primeiros e segundos pré-molares, primeiros e segundos molares, respectivamente de $6,03 \mathrm{~mm}, 9,82 \mathrm{~mm}, 8,66 \mathrm{~mm}, 9,72 \mathrm{~mm}$ e $5,67 \mathrm{~mm}$. Avaliando o comportamento dos dentes de apoio do disjuntor quanto às inclinações das coroas dentárias, notou-se que ocorreu uma vestibularização assimétrica, pois para os primeiros molares os valores encontrados foram $6,89^{\circ}$ (direito) e $9,56^{\circ}$ (esquerdo), e para os primeiros pré-molares os valores obtidos foram $4,74^{\circ}$ (esquerdo) e $3,26^{\circ}$ (direito), sendo esse considerado estatisticamente não-significativo. Conclusões: a técnica cirúrgica empregada para esta pesquisa mostrou ser eficiente para se obter alteração transversal maxilar, ocorrendo inclinação dentoalveolar dos dentes de apoio do disjuntor.

Palavras-chave: Expansão maxilar. Cirurgia ortognática. Modelos dentários.

* Baseado na tese de mestrado apresentada à Universidade Metodista de São Paulo Umesp.

** Mestre em Ortodontia pela Universidade Metodista de São Paulo (Umesp). Ortodontista do Serviço de Deformidade Craniofacial do Departamento de Cirurgia Plástica e Queimaduras da Faculdade de Medicina da Universidade de São Paulo - FMUSP.

*** Doutora em Ciências pela Unifesp. Professora titular do programa de pós-graduação em Odontologia (mestrado), área de concentração Ortodontia, da Universidade Metodista de São Paulo (Umesp).

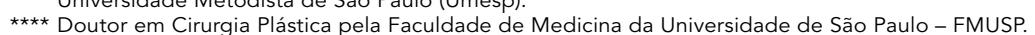

$\star \star \star \star \star$ Professor livre docente da Faculdade de Medicina da Universidade de São Paulo - FMUSP.

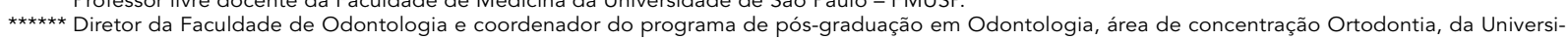
dade Metodista de São Paulo (Umesp). 


\section{INTRODUÇÃO}

As alterações transversais maxilares que levam à mordida cruzada posterior maxilar unilateral ou bilateral são responsáveis por uma série de problemas oclusais. Obter uma dimensão transversal maxilar adequada é requisito para uma oclusão funcional e estável. Na maxila, a aproximação da maturidade esquelética faz com que a interdigitação óssea aumente, como resultado da fusão das suturas craniofaciais ${ }^{11,14}$, dificultando a separação da maxila na sutura palatina mediana, se realizada somente com forças ortopédicas por meio da expansão rápida da maxila (ERM). Nesses casos, a liberação das suturas maxilares, com auxílio de osteotomias, é a forma de diminuirse a resistência óssea e tornar efetiva a expansão transversal maxilar.

Na ERMAC, é realizada uma osteotomia das estruturas que resistem às forças expansivas, trazendo vantagens, como uma expansão esquelética, ausência de dor, diminuição do risco à saúde periodontal, aumento do fluxo de ar nasal, melhora cosmética do corredor bucal, além de evitar-se extrações dentárias para correção de apinhamentos dentários ${ }^{15,16,18,20}$.

Os resultados obtidos com a expansão cirúrgica mostram um aumento significativo da largura transversal maxilar, com correção da mordida cruzada posterior e redução da profundidade do palato, minimizando a inclinação vestibular dos dentes posteriores ${ }^{13,16}$ que poderia ocorrer com a ERM.

Clinicamente, podemos observar, nos pacientes que necessitam de expansão maxilar, a presença de atresia do arco superior ou mordida cruzada posterior, cujo tratamento objetiva a sua correção.

O presente estudo tem como objetivo analisar os efeitos da ERMAC (sem a liberação da sutura pterigomaxilar), por meio de modelos de gesso, determinando as possíveis alterações transversais e inclinações vestibulares sobre os caninos, primeiros e segundos pré-molares, primeiros e segundos molares superiores, além da efetividade dessa técnica cirúrgica utilizada.

\section{MATERIAL E MÉTODOS}

A amostra utilizada para a realização deste estudo foi composta por 34 pares de modelos de gesso de 17 pacientes, sendo 6 do gênero masculino e 11 do gênero feminino, submetidos à ERMAC. Como critério de exclusão, os pacientes não poderiam apresentar má formação congênita ou síndromes faciais.

Os pacientes foram triados do Programa de Pós-Graduação em Odontologia - área de concentração Ortodontia - da Universidade Metodista de São Paulo (Umesp) e do Serviço de Cirurgia Craniomaxilofacial da Divisão de Cirurgia Plástica e Queimaduras do Instituto Central do Hospital das Clínicas da Faculdade de Medicina da Universidade de São Paulo (HCFMUSP).

Para cada paciente, foram preparados dois pares de modelos obtidos em diferentes fases: T1 - inicial (antes do procedimento operatório) - e T2 - três meses após a finalização da ativação do aparelho expansor.

O aparelho expansor utilizado foi o disjuntor do tipo Hyrax, construído com parafuso de expansão de 13mm (Morelli, Sorocaba / SP).

Cada paciente foi submetido à anestesia geral e intubação nasotraqueal. A osteotomia utilizada sobre a maxila foi do tipo Le Fort I, com separação dos pilares centrais e laterais da maxila sem o envolvimento da sutura pterigomaxilar. Foi realizada a separação da sutura palatina mediana, iniciada na espinha nasal anterior e entre os incisivos centrais sobre o osso alveolar. Após a osteotomia, o aparelho Hyrax foi ativado com o propósito de confirmar a separação da maxila, mantendo uma separação entre os incisivos centrais de $1 \mathrm{~mm}$. A ativação do aparelho iniciou-se no terceiro dia pós-operatório, sendo realizadas duas ativações durante o dia, uma no período da manhã ( $1 / 4 \mathrm{de}$ volta) e outra no período noturno (1/4 de volta) até completar a abertura total do parafuso ou atingir os objetivos oclusais ${ }^{7}$ (Fig. 1).

As medidas transversais e verticais foram realizadas em modelos de gesso posicionados em um 

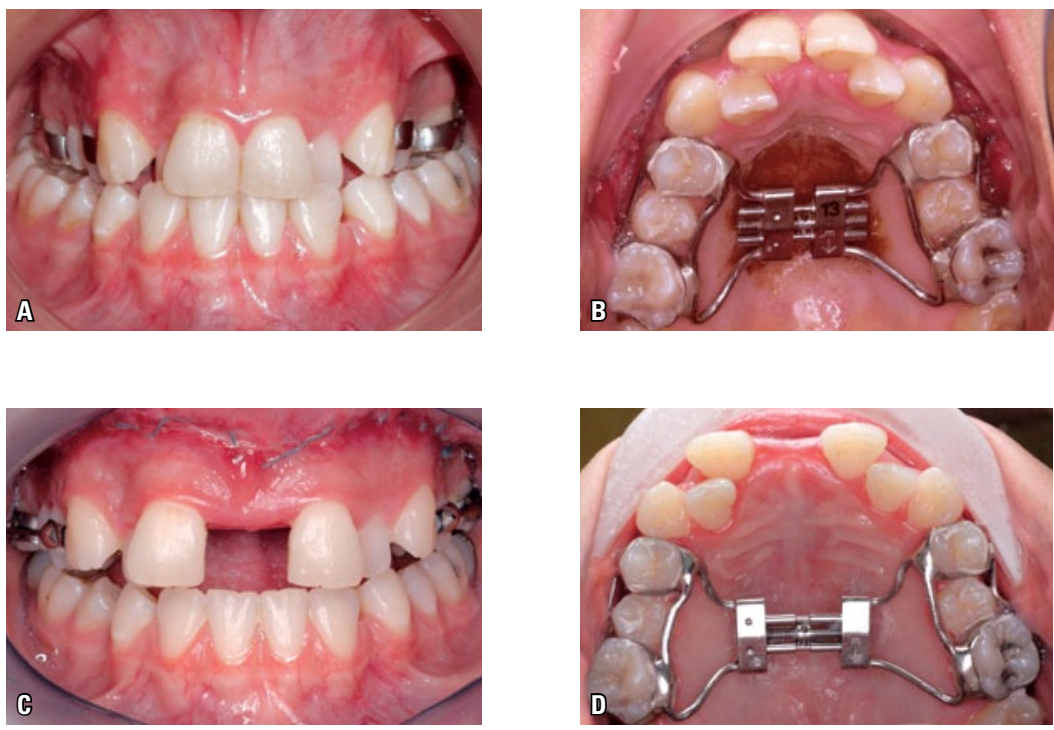

FIGURA 1 - A, B) Vistas frontal e oclusal superior do disjuntor tipo Hyrax instalado com apoios sobre os primeiros molares superiores e primeiros pré-molares, com um arco de conexão entre as bandas por vestibular e por palatino. C, D) Vistas frontal e oclusal superior do disjuntor após a ativação, demonstrando 0 efeito da expansão sobre os dentes superiores.

equipamento de medição manual tridimensional, onde os valores mensurados foram realizados com deslocamento dos três eixos, X, Y e Z (Fig. 2). O aparelho de medição foi desenvolvido sobre uma plataforma de alumínio, onde foram posicionados dois paquímetros digitais de $150 \mathrm{~mm}$, formando um ângulo de $90^{\circ}$ (plano horizontal, eixos X e Y) e um relógio comparador de medição vertical analógico de $50 \mathrm{~mm}$ (plano vertical, eixo $\mathrm{Z}$ ) da marca Mitutoyo ${ }^{\circledR}$.

A determinação dos pontos de referência no arco dentário superior foi realizada no modelo de gesso superior de cada paciente e foram demarcados pontos de referência sobre as faces oclusais dos dentes, conforme mostra a figura 3 .

As medidas transversais (eixo X) tiveram o objetivo de quantificar as alterações ocorridas após a ativação do aparelho de expansão do tipo Hyrax. As medições foram realizadas após o posicionamento do modelo de gesso na máquina de medição tridimensional, estando o plano oclusal nivelado e tendo como referência para esse nivelamento as cúspides mesiopalatinas dos primeiros molares e as bordas incisais dos incisivos centrais, sendo assim medidas as distâncias lineares entre os dentes do lado direito e esquerdo (Fig. 4).

Para a inclinação dos dentes posteriores, foram realizadas as medições das distâncias lineares horizontais (eixo $\mathrm{X}$ ) e verticais (eixo $\mathrm{Z}$ ) das cúspides vestibulares e palatinas dos primeiros pré-molares e molares superiores (Fig. 5, 6). Posteriormente, as medidas foram inseridas em uma fórmula matemática que permitiu transformar a distância linear em graus (Fig. 7). As medidas horizontais foram colocadas sobre o eixo $\mathrm{X}$ e as medidas verticais sobre o eixo $\mathrm{Z}$, como mostra a figura 6 .

Uma vez obtida a medida desejada, aplicou-se a fórmula matemática ilustrada na figura 7 , com a finalidade de determinar a quantidade de inclinação de cada dente nos períodos T1 e T2. Foi atribuído valor positivo à inclinação vestibular e negativo à inclinação palatina.

\section{RESULTADOS}

Para a verificação dos efeitos promovidos pelo tratamento, empregou-se o teste $t$ de Student com 

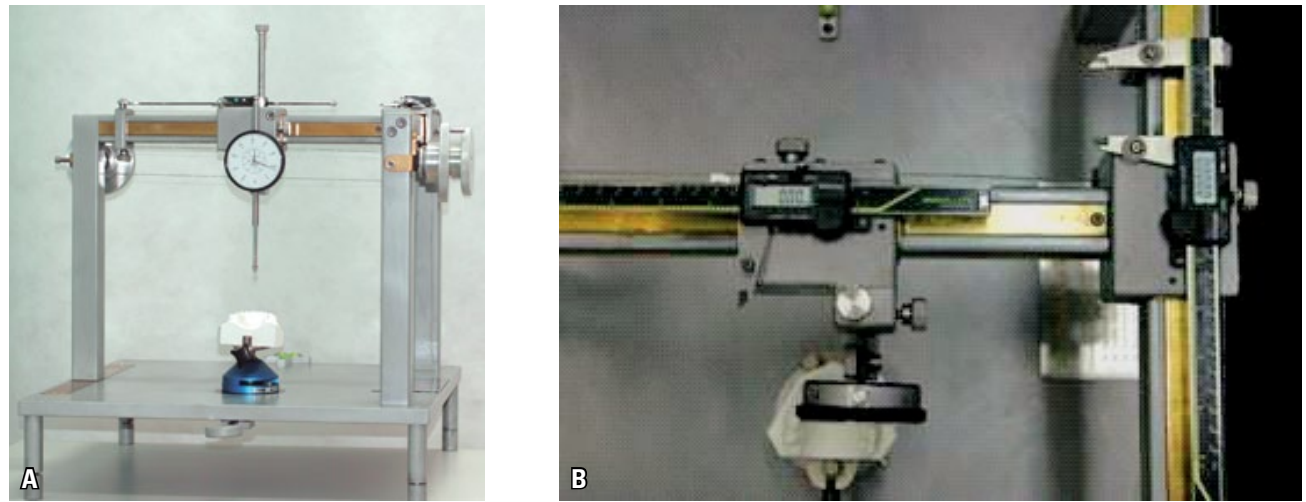

FIGURA 2 - Máquina de medição tridimensional desenvolvida na Umesp para a realização das medidas: A) vista frontal, mostrando o relógio de medição vertical (coordenada Z), e B) vista superior, mostrando os dois paquímetros digitais para as medidas transversais (coordenada X) e anteroposteriores (coordenada Y).

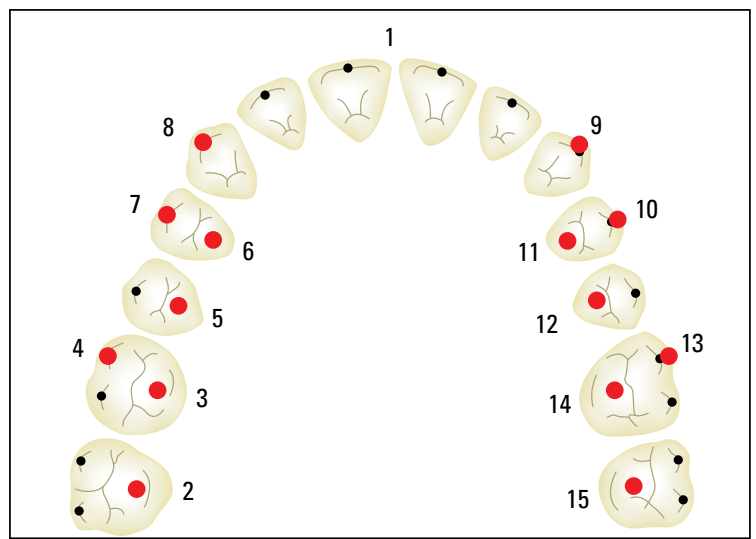

FIGURA 3 - Distâncias lineares: distância intercaninos - distância linear em mm obtida entre os pontos 8 e 9; distância interpré-molares (primeiros) - distância obtida entre os pontos 6 e 11; distância interpré-molares (segundos) - distância obtida entre os pontos 5 e 12; distância intermolares (primeiros) - distância obtida entre os pontos 3 e 14; distância intermolares (segundos) - distância obtida entre os pontos 2 e 15.

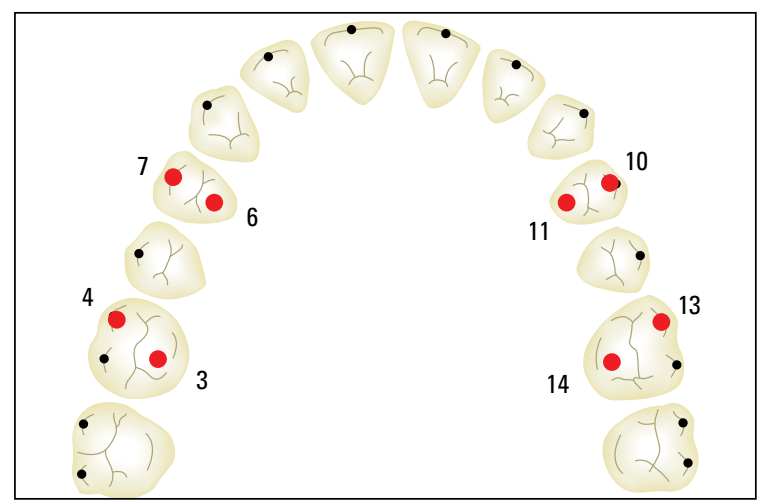

FIGURA 5 - Pontos utilizados para calcular o grau de inclinação dentária: primeiro pré-molar superior direito - pontos 7 a 6 ; primeiro pré-molar superior esquerdo - pontos 10 a 11; primeiro molar superior direito pontos 4 a 3; primeiro molar superior esquerdo - pontos 14 a 13 .

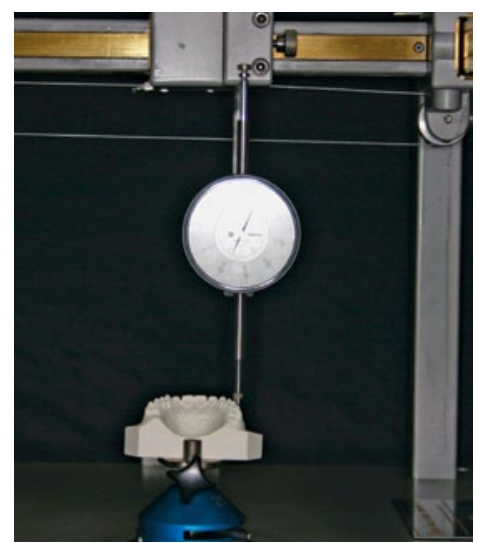

FIGURA 4 - Medidas transversais realizadas sobre os modelos de gesso.

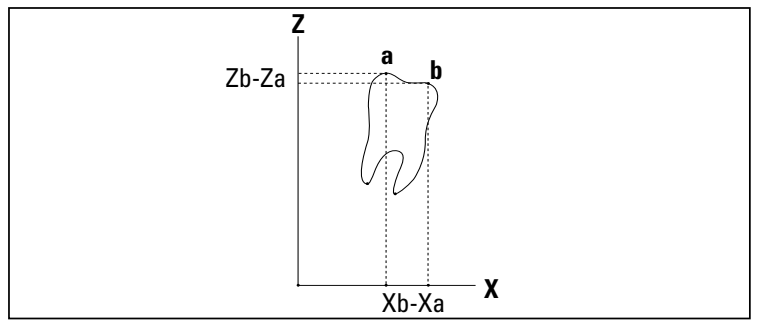

FIGURA 6 - Gráfico sobre as coordenadas $Z$ e $X$, sendo Xa (cúspide vestibular) e $\mathrm{Xb}$ (cúspide palatina) no plano horizontal, e Za (cúspide vestibular) e Zb (cúspide palatina) no plano vertical.

$$
\theta=\operatorname{arctg}\left(\frac{Z_{b}-Z_{a}}{X_{b}-X_{a}}\right) \cdot \frac{180}{\pi}
$$

FIGURA 7 - Fórmula matemática utilizada para determinar a medida angular. 
nível de significância de $5 \%(\mathrm{p}<0,05)$ para dados pareados, como consta nas tabelas 1 e 2. Para avaliação do erro sistemático, foi aplicada a fórmula de Dahlberg.

\section{Medidas transversais}

Distâncias intercaninos superiores

Houve um aumento estatisticamente significativo nas distâncias entre os caninos do lado direito e esquerdo, conforme mostra a tabela 1 . A média das diferenças (T2 - T1) entre os intervalos encontrados foi de $6,03 \mathrm{~mm}$.

\section{Distâncias interpré-molares superiores}

Houve um aumento estatisticamente significativo nas distâncias entre os primeiros e segundos pré-molares, conforme a tabela 1. A média das diferenças (T2 - T1) entre os intervalos foi de $9,82 \mathrm{~mm}$ para o primeiro pré-molar e para o segundo pré-molar foi encontrada uma variação média de $8,66 \mathrm{~mm}$.

\section{Distâncias intermolares superiores}

Houve um aumento estatisticamente significativo nas distâncias entre os primeiros e segundos molares, conforme tabela 1. A média das diferenças (T2 - T1) entre os intervalos foi de $9,72 \mathrm{~mm}$ para o primeiro molar e para o segundo molar foi encontrada uma variação média de $5,67 \mathrm{~mm}$.

\section{Inclinações dentárias}

Inclinações dos primeiros molares superiores

Após a realização das medições sobre as coordenadas $\mathrm{X}$ e $\mathrm{Z}$, conforme foi explicado nas figuras 5 e 6, e aplicada a fórmula matemática da figura 7 , pode-se observar que ocorreu inclinação dos primeiros molares superiores dos lados direito e esquerdo para vestibular, sendo os valores medidos

TABELA 1 - Distância interdentária.

\begin{tabular}{|c|c|c|c|c|c|c|c|c|}
\hline \multirow{2}{*}{ VARIÁVEIS } & \multicolumn{2}{|c|}{$\mathrm{T1}$} & \multicolumn{2}{|c|}{ T2 } & \multicolumn{2}{|c|}{ T2 - T1 } & \multirow{2}{*}{$\mathbf{T}$} & \multirow{2}{*}{$\mathbf{P}$} \\
\hline & média & d.p. & média & d.p. & média & d.p. & & \\
\hline distância intercaninos & 29,85 & 2,53 & 35,87 & 2,41 & 6,03 & 1,27 & $-18,92$ & 0,0000 \\
\hline distância inter primeiros pré-molares & 25,51 & 2,59 & 35,33 & 2,82 & 9,82 & 1,48 & $-27,33$ & 0,0000 \\
\hline distância inter segundos pré-molares & 30,96 & 3,32 & 39,62 & 4,62 & 8,66 & 5,30 & $-6,33$ & 0,0000 \\
\hline distância inter primeiros molares & 36,03 & 4,27 & 45,75 & 3,99 & 9,72 & 1,57 & $-24,74$ & 0,0000 \\
\hline distância inter segundos molares & 43,39 & 4,14 & 49,07 & 4,68 & 5,67 & 2,40 & $-9,45$ & 0,0000 \\
\hline
\end{tabular}

* diferença estatisticamente significativa $(p<0,05)$. n.s. = diferença estatisticamente não-significativa.

TABELA 2 - Inclinação vestibulolingual.

\begin{tabular}{|c|c|c|c|c|c|c|c|c|}
\hline \multirow{2}{*}{ VARIÁVEIS } & \multicolumn{2}{|c|}{ T1 } & \multicolumn{2}{|c|}{$\mathrm{T} 2$} & \multicolumn{2}{|c|}{$\mathrm{T} 2-\mathrm{T} 1$} & \multirow{2}{*}{$\mathbf{T}$} & \multirow{2}{*}{$\mathbf{P}$} \\
\hline & média & d.p. & média & d.p. & média & d.p. & & \\
\hline inclinação molar direito & 7,28 & 8,02 & 14,17 & 7,18 & 6,89 & 5,55 & $-5,12$ & 0,0001 \\
\hline inclinação molar esquerdo & 4,01 & 9,48 & 13,57 & 9,45 & 9,56 & 9,08 & $-4,34$ & 0,0005 \\
\hline inclinação pré-molar direito & $-5,58$ & 10,11 & $-2,32$ & 7,97 & 3,26 & 6,96 & $-1,93$ & 0,0715 \\
\hline inclinação pré-molar esquerdo & $-7,33$ & 7,37 & $-2,58$ & 7,68 & 4,74 & 3,98 & $-4,92$ & 0,0002 \\
\hline
\end{tabular}

* diferença estatisticamente significativa $(p<0,05)$. n.s. = diferença estatisticamente não-significativa. 
estatisticamente significativos, conforme mostra a tabela 2. A variação observada, em média, foi de $6,89^{\circ}$, mostrando uma inclinação vestibular do primeiro molar direito. Para o primeiro molar esquerdo, foi observada uma inclinação vestibular média de $9,56^{\circ}$.

Inclinações dos primeiros pré-molares superiores

O comportamento dos primeiros pré-molares não foi estatisticamente similar, pois mostrou uma tendência à inclinação assimétrica. Nos primeiros pré-molares do lado direito, as inclinações encontradas não foram consideradas estatisticamente significativas, conforme mostra a tabela 2. Porém a inclinação vestibular medida para os primeiros pré-molares do lado esquerdo mostrou-se estatisticamente significativa, conforme mostra a tabela 2 , cuja variação encontrada foi de $4,74^{\circ}$.

\section{DISCUSSÃO}

Timms e Vero ${ }^{21}$ sugerem que, em pacientes com idade inferior a 25 anos, deve-se tentar a expansão ortopédica da maxila ${ }^{8}$ (ERM). Porém, existe risco de falhas nesse tipo de tratamento, bem como também deve-se considerar o alto índice de recidiva em adultos ${ }^{2,4,10,14,22}$.

A fusão das articulações maxilares é o fator responsável por essa dificuldade. Desse modo, a escolha da técnica cirúrgica com liberação de todas as articulações maxilares é relatada na literatura como sendo a mais adequada, pois, como afirmam Betts et $\mathrm{al}^{7}{ }^{7}$, a liberação do processo pterigoide deve ser realizada para que não ocorra uma limitação na expansão maxilar no segmento posterior. Kennedy et al. ${ }^{12}$ realizaram estudos em macacos e concluíram que o pilar zigomático é a área que oferece maior resistência à expansão maxilar, porém, para que ocorra o movimento basal da maxila, todos os pilares e articulações maxilares devem ser seccionados. Assim, a escolha da área da osteotomia é assunto de muita discussão e controvérsia. Baseado em achados da literatura que mostram a efetividade dos resultados da expansão em pacientes submetidos a osteotomias maxilares ${ }^{1,3,14,17}$, a técnica cirúrgica selecionada para o presente trabalho não envolveu a separação do processo pterigoide durante a osteotomia. A quantidade de expansão obtida deve ser correlacionada com a quantidade de inclinação vestibular, para se determinar a efetividade da técnica cirúrgica. Poucos trabalhos correlacionam expansão e inclinação dentoalveolar.

A quantidade de alteração transversal produzida pela ERMAC é efetiva tanto na região anterior quanto na região posterior da maxila ${ }^{1,3,6,9,14,17,19}$. Na tabela 3 , pode-se avaliar a efetividade desse procedimento, em artigos encontrados na literatura.

A comparação dos resultados obtidos na presente amostra com os de outros artigos evidencia que a quantidade de expansão obtida foi adequada com os achados da literatura, apesar da técnica cirúrgica não envolver a osteotomia da sutura pterigomaxilar.

Avaliando a alteração transversal entre os caninos, o resultado obtido na amostra evidencia que ocorreu um aumento nas distâncias entre os caninos do lado direito e esquerdo estatisticamente significativo. A média encontrada para as diferenças (T2 - T1) entre os intervalos foi de $6,03 \mathrm{~mm}$. Comparando com outros achados, percebe-se que foi obtida uma variação importante sobre os caninos e pode-se perceber que a variação entre os trabalhos analisados foi de $4,1 \mathrm{~mm}$ e $6,03 \mathrm{~mm}$ mostrando que, independentemente da técnica cirúrgica utilizada, foram obtidas variações transversais significativas para esses elementos dentários, apesar dos mesmos não receberem apoio direto por meio de bandas ou fios.

A distância transversal entre os pré-molares superiores mostrou um aumento estatisticamente significativo. Para os primeiros pré-molares, a média entre os intervalos encontrados foi de $9,82 \mathrm{~mm}$; e, para os segundos pré-molares, foi de $8,66 \mathrm{~mm}$. Pode-se perceber que a variação foi similar entre os primeiros e os segundos pré-molares, 
TABELA 3 - Comparação de artigos que realizaram mensurações das alterações dentárias em pacientes submetidos à ERMAC.

\begin{tabular}{|c|c|c|c|c|c|c|}
\hline AUTOR & OSTEOTOMIA & CANINOS & $\begin{array}{l}\text { PRIMEIROS } \\
\text { PRÉ-MOLARES }\end{array}$ & $\begin{array}{l}\text { SEEUNDOS } \\
\text { PRÉ-MOLARES }\end{array}$ & $\begin{array}{l}\text { PRIMEIROS } \\
\text { MOLARES }\end{array}$ & $\begin{array}{l}\text { SEGUNDOS } \\
\text { MOLARES }\end{array}$ \\
\hline Anttila et al. ${ }^{1}$ & $\begin{array}{l}\text { osteotomia na parede } \\
\text { lateral maxilar }\end{array}$ & $4,1 \mathrm{~mm}$ & $6,8 \mathrm{~mm}$ & $7,2 \mathrm{~mm}$ & $7,2 \mathrm{~mm}$ & $5,1 \mathrm{~mm}$ \\
\hline Bays, Grecco ${ }^{3}$ & $\begin{array}{l}\text { osteotomia na parede } \\
\text { lateral maxilar }\end{array}$ & $4,5 \mathrm{~mm}$ & 0 & 0 & $5,8 \mathrm{~mm}$ & 0 \\
\hline Berger et al. ${ }^{6}$ & $\begin{array}{l}\text { osteotomia Le Fort com } \\
\text { envolvimento da sutura } \\
\text { pterigomaxilar }\end{array}$ & $4,84 \mathrm{~mm}$ & 0 & 0 & $5,78 \mathrm{~mm}$ & 0 \\
\hline Byloff, Mossaz ${ }^{9}$ & $\begin{array}{l}\text { osteotomia Le Fort com } \\
\text { envolvimento da sutura } \\
\text { pterigomaxilar }\end{array}$ & $5,19 \mathrm{~mm}$ & $8,08 \mathrm{~mm}$ & $8,26 \mathrm{~mm}$ & $8,73 \mathrm{~mm}$ & $5,48 \mathrm{~mm}$ \\
\hline Northway, Meade ${ }^{14}$ & $\begin{array}{c}\text { osteotomia nas paredes } \\
\text { laterais }\end{array}$ & $4,26 \mathrm{~mm}$ & 0 & 0 & $5,9 \mathrm{~mm}$ & 0 \\
\hline Schimming et al. ${ }^{17}$ & $\begin{array}{c}\text { osteotomia nas paredes } \\
\text { laterais }\end{array}$ & $5,9 \mathrm{~mm}$ & 0 & 0 & $6,0 \mathrm{~mm}$ & 0 \\
\hline Stromberg, Holm 19 & $\begin{array}{l}\text { osteotomia Le Fort com } \\
\text { envolvimento da sutura } \\
\text { pterigomaxilar }\end{array}$ & $5,0 \mathrm{~mm}$ & 0 & 0 & $8,3 \mathrm{~mm}$ & 0 \\
\hline
\end{tabular}

mostrando que o segundo pré-molar acompanhou o movimento transversal produzido. A diferença de $1,16 \mathrm{~mm}$ entre os primeiros e os segundos pré-molares se deve, provavelmente, à ausência de apoios de bandas sobre os segundos pré-molares, que contou com um apoio por palatino (fio de aço). Os valores obtidos na literatura foram similares, porém Antilla et al. ${ }^{1}$, Byllof e Mossaz ${ }^{9}$ encontraram uma aumento da distância transversal maior no segundo pré-molar, conforme mostra a tabela 3, comportamento esse diferente do presente estudo e dos demais artigos da literatura.

Avaliando a distância transversal dos molares, a média entre os intervalos encontrada para os primeiros molares foi de $9,72 \mathrm{~mm}$ e para os segundos molares obteve-se uma variação de $5,67 \mathrm{~mm}$. O valor encontrado entre os primeiros pré-molares e primeiros molares mostrou um comportamento similar, porém deve-se lembrar que foram os dentes que serviram de apoio para a aplicação das forças expansoras e que também estavam conectados, por meio de bandas, ao parafuso expansor. Tal comportamento é observado em outros trabalhos.
Porém, a quantidade de alteração transversal obtida nos segundos molares chamou a atenção, os quais responderam com uma variação menor, também observada em outros trabalhos.

Pode-se dizer que tal variação seja devida ou à falta de uma extensão da osteotomia sobre a sutura pterigomaxilar ou à falta de um apoio direto sobre os segundos molares. No trabalho de Byloff e Mossaz $^{9}$, que utilizaram osteotomia mais invasiva, o resultado encontrado para o segundo molar é similar ao obtido na presente amostra. Sendo assim a osteotomia não deve ser responsável pela falta de expansão nessa área, devida à falta de apoio por meio de bandas nos segundos molares, o que levou a uma menor alteração transversal. Comparando a medida encontrada para os caninos $(6,03 \mathrm{~mm})$ e segundos molares $(5,67 \mathrm{~mm})$, percebe-se que há uma correlação entre os valores encontrados, visto que ambos os grupos não receberam apoio direto do disjuntor durante a ativação. Essa alteração pode ser resultado da mudança que ocorreu sobre o osso da maxila somente.

Quanto às inclinações observadas nos dentes 
posteriores, os resultados observados mostraram um comportamento assimétrico para esse grupo de dentes, onde os elementos dentários do lado esquerdo apresentaram uma inclinação maior para vestibular, podendo ser uma característica da amostra analisada. A metodologia empregada para essa amostra permite medir individualmente a quantidade de inclinação que cada elemento dentário sofreu.

As alterações dos primeiros molares superiores direito e esquerdo foram calculadas nos intervalos de tempo T1 e T2, com a finalidade de quantificar a movimentação ocorrida. Os valores encontrados foram estatisticamente significativos, conforme mostra a tabela 2 . A variação média observada foi de $6,89^{\circ}$, mostrando uma significativa inclinação para vestibular no molar do lado direito. Para o molar do lado esquerdo, foi observada uma inclinação média de $9,56^{\circ}$ para vestibular. Os resultados observados mostraram um comportamento assimétrico para esse grupo de dentes, sendo que os dentes do lado esquerdo apresentaram uma inclinação estatisticamente maior para vestibular, podendo ser uma característica da amostra analisada.

As alterações encontradas nos primeiros prémolares superiores direito e esquerdo nos intervalos de tempo T1 e T2 mostraram comportamentos diferentes, conforme a tabela 2. Para o lado direito, a variação média observada foi de $3,26^{\circ}$, estatisticamente não-significativa. Porém, a inclinação vestibular medida para os primeiros pré-molares do lado esquerdo mostrou-se estatisticamente significativa, com variação de $4,74^{\circ}$. A inclinação medida para esses dentes também mostrou um comportamento assimétrico quanto à movimentação vestibular dos dentes posteriores.

A amostra demonstrou que existe uma efetividade para o ganho transversal, porém ocorreu movimento pendular, para vestibular, das coroas dos dentes de apoio do aparelho Hyrax. Os dentes que mostraram maiores mudanças transversais foram os primeiros pré-molares e os primeiros molares superiores. As alterações sobre esses dentes são resultado da composição de dois efeitos: o movimento expansivo transversal que ocorre em decorrência do afastamento ósseo e a inclinação das coroas dos dentes para vestibular. A inclinação vestibular poderá ser considerada um efeito indesejável, de acordo com a necessidade clínica de cada paciente, pois o movimento lateral dos dentes desejável deveria ser mais paralelo.

\section{CONCLUSÕES}

Pode-se concluir, diante dos resultados obtidos com a técnica cirúrgica utilizada na ERMAC, que:

- Quanto às alterações transversais, houve um aumento estatisticamente significativo nas distâncias transversais na região dos caninos, primeiros e segundos pré-molares, primeiros e segundos molares.

- Houve um aumento estatisticamente significativo nas inclinações dos primeiros molares dos lados direito e esquerdo, e dos primeiros pré-molares de um dos lados, sugerindo um comportamento assimétrico dos dentes avaliados a partir da ERMAC.

- A técnica cirúrgica utilizada mostrou-se efetiva, pois permitiu que ocorressem alterações transversais na maxila, porém com inclinação para vestibular das coroas dos dentes de apoio do aparelho expansor. 


\title{
Assessment of maxillary dental changes in patients submitted to surgically assisted rapid maxillary expansion with no involvement of pterygoid blade
}

\begin{abstract}
Aim: To assess the changes to the superior dental arches, in the transversal direction, and the amount of inclinations in the support teeth of the Hyrax expansion appliance in patients who underwent surgically assisted rapid maxillary expansion (SARME), as well as the effectiveness of the surgical technique used. The sample was composed of 34 pairs of cast models, of 17 patients -6 males and 11 females. Methods: The measurements were performed on cast models, by measuring the changes on the vertical and transversal planes. The surgical procedure used was the lateral osteotomy on maxillary walls with no involvement of the pterygoid blade, osteotomy of nasal spine on dental midline (anterior central incisors), chisel separation of palatine suture, and separation of the nasal septum. The activation was initiated on the third postoperative day, consisting of two activations, one in the morning and another at night. Results: There were statistically significant expansions in the regions of canines, first and second premolars, first molars, and second molars; respectively, $6.03 \mathrm{~mm}, 9.82 \mathrm{~mm}, 8.66 \mathrm{~mm}, 9.72 \mathrm{~mm}$, and $5.67 \mathrm{~mm}$. The evaluation of the behavior of the support teeth of the expansion appliance regarding the inclination of the dental crowns has shown that an asymmetric vestibularization had taken place, because the values found for the first molars were $6.89^{\circ}$ (right) and $9.56^{\circ}$ (left); the values for the first premolars were $4.74^{\circ}$ (left) and $3.26^{\circ}$ (right); this last value was not statistically significant. Conclusions: The surgical technique used in this research has been shown to be efficient to obtain maxillary transversal alterations, and there was dental-alveolar inclination of the support teeth of the expansion appliance.
\end{abstract}

Keywords: Maxillary expansion. Orthognathic surgery. Dental casts.

\section{REFERÊNCIAS}

1. ANTTILA, A. et al. Feasibility and long term stability of surgically assisted rapid maxillary expansion with lateral osteotomy. Eur. J. Orthod., Oxford, v. 26, no. 4, p. 391-395, 2004.

2. BARBER, A. F.; SIMS, M. R. Rapid maxillary expansion and external root resorption in man: A scanning microscope study. Am. J. Orthod., St. Louis, v. 79, no. 6, p. 630-652, June 1981.

3. BAYS, R. A.; GRECO, J. M. Surgically assisted rapid palatal expansion: An outpatient technique with long-term stability. J. Oral. Maxillofac. Surg., Stuttgart, v. 50, no. 2, p. 110-113, Feb. 1992.

4. BELL, R. A. A review of maxillary expansion in relation to rate of expansion and patient's age. Am. J. Orthod., St. Louis, v. 81, no. 1, p. 32-37, Jan. 1982.

5. BELL, W. H.; EPKER, B. N. Surgical-orthodontic expansion of the maxilla. Am. J. Orthod. Dentofacial Orthop., St. Louis, v. 70, no. 5, p. 517-528, Nov. 1976.

6. BERGER, J. L. et al. Stability of orthopedic and surgically assisted rapid palatal expansion over time. Am. J. Orthod. Dentofacial Orthop., St. Louis, v. 114, no. 6, p. 638-645, Dec. 1998.

7. BETTS, N. J. et al. Diagnosis and treatment of transverse maxillary deficiency. J. Adult Orthod. Orthognat. Surg., Chicago, v. 10, no. 2, p. 75-96, June 1995.

8. BIEDERMAN, W. A hygienic appliance for rapid expansion. JPO: J. Pract. Orthod., Hempstead, v. 2, no. 2, p. 67-70, Feb. 1968.

9. BYLOFF, F. K.; MOSSAZ, C. F. Skeletal and dental changes following surgically assisted rapid palatal expansion. Eur. J. Orthod., Oxford, v. 26, no. 4, p. 403-409, July/Aug. 2004.

10. CAPELOZZA FILHO, L.; CARDOSO NETO, J.; SILVA FILHO, O. G.; URSI, W. J. S. Non-surgically assisted rapid maxillary expansion in adults. Int. J. Adult Orthodon. Orthognath. Surg., Chicago, v. 11, no. 1, p. 57-66, 1996.

11. GURGEL, J. A.; SANT'ANA, E.; HENRIQUES, J. F. C. Tratamento orto-cirúrgico das deficiências transversais da maxila.

R. Dental Press Ortodon. Ortop. Facial, Maringá, v. 6, n. 6, p. 59-66, nov./dez. 2001.

12. KENNEDY, J. W.; BELL, W. H.; KIMBROUGH, O. L.; BARRY, J.

W. Osteotomy as adjunct to rapid maxillary expansion. Am. J. Orthod. Dentofacial Orthop., St. Louis, v. 70, no. 2, p. 123-137, Apr. 1976
13. LINES, P. A. Adult rapid maxillary expansion with corticotomy. Am. J. Orthod. Dentofacial Orthop., St. Louis, v. 67, no. 1, p. 44-56, 1975.

14. NORTHWAY, W. M.; MEADE JR., J. B. Surgically assisted rapid maxillary expansion: A comparison of technique, response, and stability. Angle Orthod., Appleton, v. 67, no. 4, p. 309-320, 1997

15. PERSSON, M.; THILANDER, B. Palatal suture closure in man from 15 to 35 years of age. Am. J. Orthod. Dentofacial Orthop., St. Louis, v. 72, no. 1, p. 42-52, 1977.

16. RIBEIRO JÚNIOR, P. D. et al. Avaliação clínica dos procedimentos de expansão cirurgicamente assistida da maxila (ECAM). R. Dental Press Ortodon. Ortop. Facial, Maringá, v. 11, n. 1, p. 44-59, jan./fev. 2006

17. SCHIMMING, R. et al. Surgically and orthodontic rapid palatal expansion in adults using Glassman's technique: Retrospective study. Br. J. Oral Surg., Edinburgh, v. 38, no. 1, p. 66-69, June 2000.

18. SILVERSTEIN, K.; QUINN, P. D. Surgically-assisted rapid palatal expansion for management of transverse maxillary deficiency. J. Oral. Maxillofac. Surg., Philadelphia, v. 55, no. 7, p. 725-727, 1997.

19. STROMBERG, C.; HOLM, J. Surgically assisted rapid maxillary expansion in adults: A retrospective long-term follow-up study. J. Craniomaxillofac. Surg., Edinburgh, v. 23, no. 4, p. 222-227, 1995.

20. TIMMS, D. J. et al. A study of basal movement with rapid maxillary expansion. Am. J. Orthod. Dentofacial Orthop., St. Louis, v. 77, no. 5, p. 500-507, May 1980.

21. TIMMS, D. J.; VERO, D. The relationship of rapid maxillary expansion to surgery with special reference to midpalatal synostosis. Br. J. Oral Surg., Edinburgh, v. 19, no. 3, p. 180-196, 1981

22. TURVEY, T. A. Maxillary expansion: A surgical technique based on surgical-orthodontic treatment objectives and anatomical considerations. J. Maxilofac. Surg., Stuttgart, v. 13, no. 2, p. 51-58, Apr. 1985.

Endereço para correspondência

Paulo Roberto Pelucio Camara

Rua Apeninos 930 cj. 93, Paraíso

CEP: 04.104-020 - São Paulo / SP

E-mail: prpcamara@uol.com.br 